\title{
Free Space based Hollow Core Fiber Interconnection and Associated In-Line Components
}

\author{
Hyuntai Kim,* Yongmin Jung,** Yong Chen, Shuichiro Rikimi, Francesco Poletti, and David J. Richardson \\ Optoelectronics Research Centre, University of Southampton, Southampton, SO17 1BJ, UK \\ *h.kim@soton.ac.uk and**ymj@orc.soton.ac.uk
}

\begin{abstract}
We present compact, low-loss optical interconnection devices based on micro-collimator technology for hollow core fibers. Exemplar functional optical components (i.e. isolator and bandpass filter) are fabricated with low backreflection $(<-47 \mathrm{~dB})$ and high modal purity $(>20 \mathrm{~dB})$. OCIS codes: (060.4005) Microstructured fibers; (060.2340) Fiber optics components;
\end{abstract}

\section{Introduction}

Hollow core fibers (HCFs), which guide light within a hollow air region, have attracted great attention as a promising means to overcome many of the limitations associated with conventional solid core fibers. Various types of HCFs (e.g. photonic bandgap fibers, tubular fibers, anti-resonant fibers) have been proposed to exploit the fascinating features of guidance in an air core (e.g. low nonlinearity, low latency, high thermal stability and high radiation hardness) and such fibers have shown excellent performance in applications such as high power laser beam delivery, optical communications, gyroscopes, and Raman spectroscopy [1-3]. However, whilst such fibers have been available for several years, there is still a lack of reliable interconnection techniques for HCF technology. Fusion splicing is the most widely used approach to interconnect a HCF to a conventional single mode fiber (SMF) but unless other steps are taken this results in a Fresnel reflection (typically $\sim 4 \%$ ) at the air-glass interface which can be problematic for many applications. To suppress the Fresnel back reflections, angle-cleaved fiber splicing (i.e. angle cleaving for both HCF and solid core fiber) has been proposed [4] but the output beam direction from the angle-cleaved solid fiber is deflected from the fiber axis, resulting in increased splice loss and the excitation of higher-order HCF modes. In addition, the mode field diameters (MFDs) of the HCFs are larger than those of typical SMFs and appropriate mode field adaptation technique are required to minimize loss and for high mode purity due to MFD mismatch [5].

In this paper, we propose and demonstrate a compact air gap device using a micro-collimator technology for SMFHCF and HCF-HCF interconnections. Such a platform has previously been widely used for the development of many (solid fiber coupled) optical components $[6,7]$ and we further investigate use of micro-collimator technology with HCFs. Several practical (and stable) exemplar in-line HCF-integrated components are demonstrated with low insertion loss, good modal quality, and low Fresnel back reflections.

\section{SMF to HCF interconnection}

The HCF used in the devices/experiments described hereafter is a 19-cell hollow-core photonic bandgap fiber (PBGF). The scanning electron microscope (SEM) image of the fiber cross-section and attenuation spectrum are shown in Fig. $1(\mathrm{a}, \mathrm{b})$ respectively. The fiber has a core diameter of $30 \mu \mathrm{m}$, a microstructured cladding diameter of $84 \mu \mathrm{m}$. The fiber loss is $\sim 3.9 \mathrm{~dB} / \mathrm{km}$ at $1550 \mathrm{~nm}$. In order to characterize the modal properties of the PBGF, the impulse response of a $350 \mathrm{~m}$ length was examined with a time-of-flight (ToF) measurement method [8]. The SMF28 output fiber from a $1550 \mathrm{~nm}$ femtosecond laser source was butt coupled to the PBGF (using a commercial fusion splicer for alignment). As shown in Fig. 1(c), when two fibers were aligned on-axis (i.e. central alignment), the $\mathrm{LP}_{02}$ mode content (5.5 ns delay and $-12.7 \mathrm{~dB}$ power relative to than $\mathrm{LP}_{01}$ ) was the dominant high order mode $(\mathrm{HOM})$ due to the nature of the modal symmetry. For off-axis alignment, however, we can observe other asymmetric higher-order modes (1.8-2.3 ns for $\mathrm{LP}_{11}$ and $4.2-4.8 \mathrm{~ns}$ for $\mathrm{LP}_{21}$ ) in this fiber.

(a)

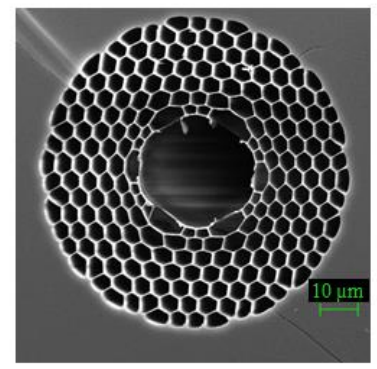

(b)

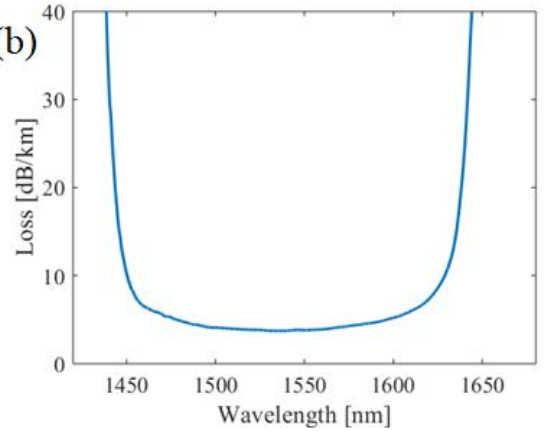

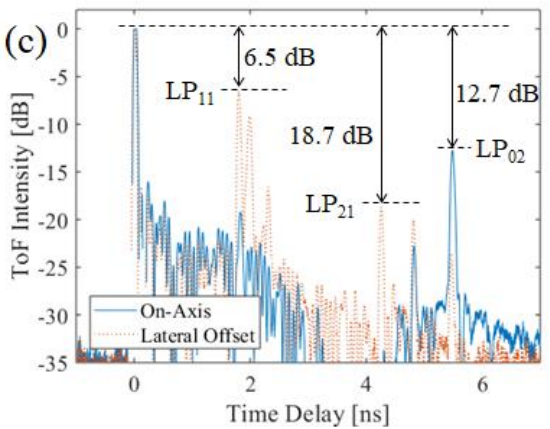

Fig. 1. (a) Cross-section SEM image and (b) fiber attenuation spectrum of the PBGF. (c) ToF measurement over 350m of PBGF. 
Figure 2(a) shows a schematic of our SMF-PBGF interconnection device. Using micro-optic collimators (diameter $=1.8 \mathrm{~mm}$ ), light emerging from the input fiber was collimated in free space and then coupled in to the output fiber $[6,7]$. Note that a large mode area fiber (LMAF) having similar a MFD to our PBGF was used as an input fiber and a mode field adaptor (MFA) was incorporated to reduce the splice loss between the SMF and LMAF [9]. Importantly, the end of the LMAF was angle-cleaved with an angle of $8^{\circ}$ to eliminate the Fresnel back reflection. The interconnection loss from the SMF input to PBGF output was $0.53 \mathrm{~dB}$ - where the loss contribution from the SMFLMAF connection with MFA was $0.28 \mathrm{~dB}$ and the loss from the LMAF-HCF micro-collimator device was $0.25 \mathrm{~dB}$. The measured back reflection was $-49.8 \mathrm{~dB}$. We also tested the mode quality after the device using a ToF method and less than $-20 \mathrm{~dB}$ of higher order mode content was observed compared to the fundamental mode $\left(-21.2 \mathrm{~dB}\right.$ for $\mathrm{LP}_{11}$ and $-22.1 \mathrm{~dB}$ for $\left.\mathrm{LP}_{02}\right)$. Note that this value is much better than the previous SMF-PBGF butt coupled joint $(-12.7 \mathrm{~dB}$ in Fig. 1(c)) due to the better match between the MFDs. Importantly, the current mode quality could be further improved by placing another micro-collimator assembly at the output end of the PBGF (i.e. an identical device but for the interconnection from PBGF to SMF).
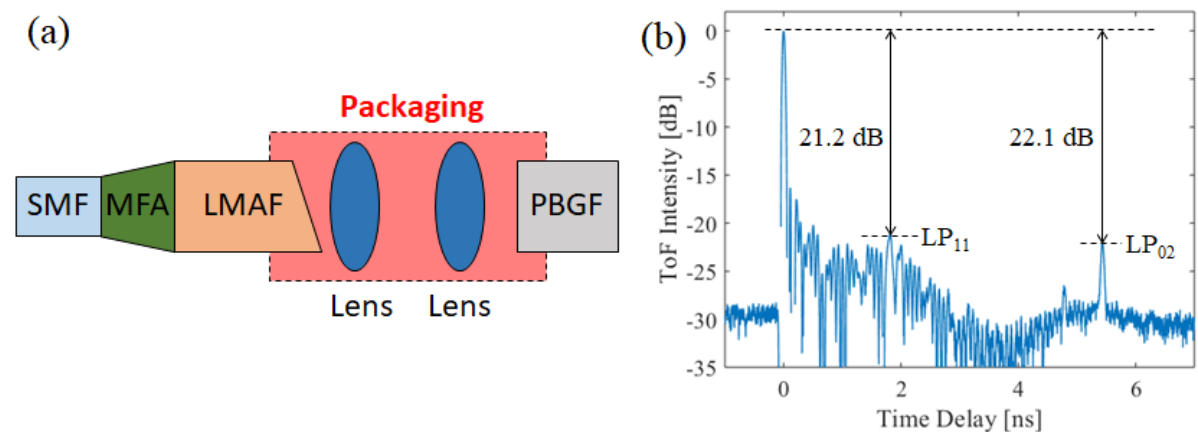

Fig. 2. (a) Schematic of the SMF-PBGF interconnection device and (b) ToF measurement from the device.

In order to realize a practical HCF device, a functional element (e.g. isolator, filter, polarizer, etc.) can be placed in the air-gap between the two micro-collimators. Here, we have implemented an optical isolator inside the device. The total device loss was $1.5 \mathrm{~dB}$ and the back reflection was $-47 \mathrm{~dB}$. The isolation spectrum is shown in Fig. 3(a) and the maximum isolation is measured to be $>40 \mathrm{~dB}$ at $1550 \mathrm{~nm}$. The devices are packaged in a $12 \mathrm{~mm} \times 9 \mathrm{~mm} \times 70 \mathrm{~mm}$ sized aluminum box as shown in Fig. 3(b). The characteristics of the basic SMF-PBGF air-gap device are summarized in Fig. 3(c), where the properties of the interconnection device are shown above the dashed line and the properties of the SMF-PBGF isolator are shown below. It is also worth noting that we have tested the long-term stability of the device for 4 months and no performance degradation was observed due to the near hermetic seal obtained from the glass mounting tubes and UV curable epoxies used in packaging the device.
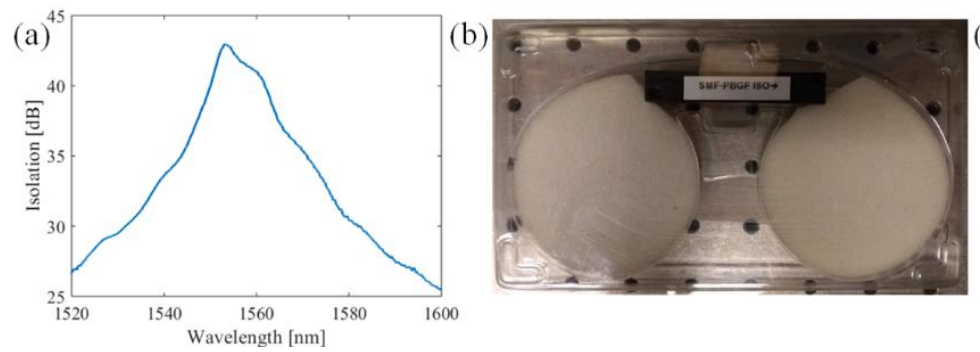

\begin{tabular}{l|l}
\hline Parameter & Value \\
\hline Fiber type & SMF / PBGF \\
Operation wavelength & $1550 \mathrm{~nm}$ \\
Insertion loss & $0.53 \mathrm{~dB}$ \\
Higher order mode intensity & $<-20 \mathrm{~dB}$ \\
Back reflection & $-49.8 \mathrm{~dB}$ \\
\hline Insertion loss (Isolator) & $1.5 \mathrm{~dB}$ \\
Isolation @ $1550 \mathrm{~nm}$ & $40.2 \mathrm{~dB}$ \\
\hline
\end{tabular}

Fig. 3. (a) Isolation spectrum of SMF-PBGF isolator. (b) Photograph of the packaged device. (c) Summary specification of SMF-PBGF device.

\section{HCF to HCF interconnection}

HCF to HCF interconnection is less problematic compared to SMF-HCF connection, because it represents a simple interconnection between two identical fibers and the Fresnel back reflections are negligible as there are no glass/air interfaces (except for anti-reflection coated micro-lenses). The schematic of the HCF-HCF device is shown in Fig. 4(a). Two identical PBGFs were used as input/output fibers and the SMF-PBGF interconnection device fabricated in the previous section was used to launch light from the SMF to PBGF. The PBGF-PBGF device loss was measured to be $\sim 1.28 \mathrm{~dB}$ and good modal purity was observed as shown in Fig. 4(b) (the intensity of the higher-order modes was less than $-20 \mathrm{~dB}$ relative to the fundamental). 
(a)

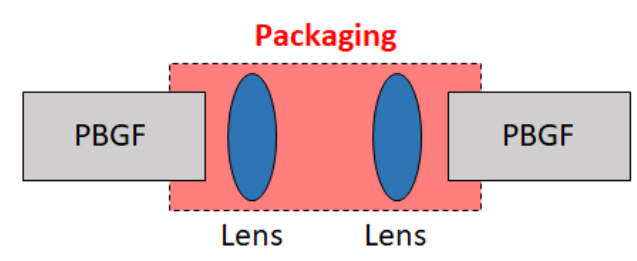

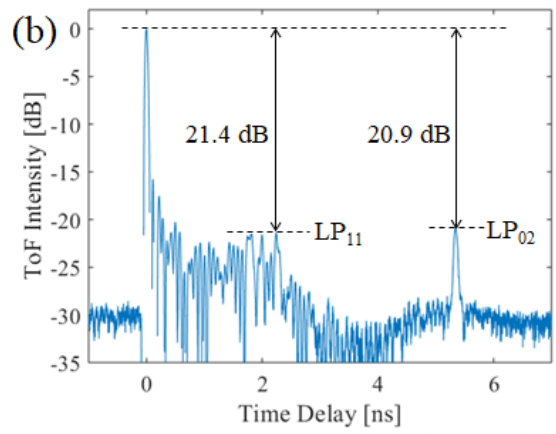

Fig. 4. (a) Schematic of the HCF-HCF interconnection device and (b) ToF measurement of the device.

To provide a functional device example a band pass filter (BPF) chip ( $3 \mathrm{~dB}$ bandwidth $\sim 7 \mathrm{~nm}$, center wavelength $=1548 \mathrm{~nm}$ ) was inserted within the air gap of the PBGF-PBGF interconnection assembly. The total device loss was $1.85 \mathrm{~dB}$, including the insertion loss of the BPF chip itself. The measured optical spectra before and after the BPF is shown in Fig. 5(a). A PBGF coupled flat-top single-band BPF was obtained with a minimum passband-torejection band ratio of $60 \mathrm{~dB}$. The specification of the PBGF-PBGF interconnection device is summarized in Fig. 5(b) (the properties above the dashed lines are for the interconnection device and under the dashed lines are for the PBGFPBGF BPF).

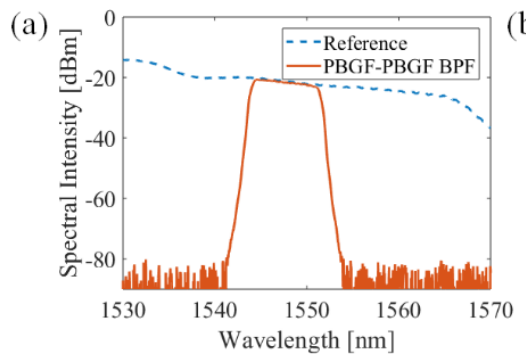

\begin{tabular}{|c|c|}
\hline Parameter & Value \\
\hline Fiber type & PBGF \\
\hline Operation wavelength & $1550 \mathrm{~nm}$ \\
\hline Insertion loss & $1.28 \mathrm{~dB}$ \\
\hline Higher order mode intensity & $<-20 \mathrm{~dB}$ \\
\hline Insertion loss (BPF) & $1.85 \mathrm{~dB}$ \\
\hline Center wavelength & $1548 \mathrm{~nm}$ \\
\hline 3 dB bandwidth & $7 \mathrm{~nm}$ \\
\hline
\end{tabular}

Fig. 5. (a) Measured optical spectra before and after the BPF and (b) summary specification of PBGF-PBGF device.

\section{Conclusion}

We have proposed and fabricated several low-loss and free-spaced based approaches to interconnect hollow core fibers. A pair of compact micro-lenses were used to collimate the light from the input fiber and to focus it into the output fiber. For the SMF-HCF interconnection, a very small insertion loss $(\sim 0.53 \mathrm{~dB})$ was achieved with a low back reflection $(<-47 \mathrm{~dB})$ and high modal purity $(>20 \mathrm{~dB})$. An optical isolator was realized as a functional device, and the total device loss remained less than $1.5 \mathrm{~dB}$, with an optical isolation of $>40 \mathrm{~dB}$ at $1550 \mathrm{~nm}$. For the HCF-HCF interconnection, an insertion loss of $\sim 1.28 \mathrm{~dB}$ with a modal purity of $>20 \mathrm{~dB}$ was achieved, and a BPF was fabricated as an example of a practical component. This compact, stable and low-loss interconnection platform for HCFs can be further optimized and integrated within more complex devices/systems and promises to be a valuable technology in support of various aspects of HCF related research.

\section{Acknowledgements}

This work was supported by the UK ESPRC funded “Airguide Photonics” Programme Grant (EP/P030181/1).

\section{References}

[1] F. Poletti et al., "Toward high-capacity fibre-optic communications at the speed of light in vacuum," Nat. Photonics 7(4), 279-284 (2013).

[2] F. Benabid et al., "Stimulated Raman scattering in hydrogen-filled hollow-core photonic crystal fiber," Science 297(5592), 399-402 (2002).

[3] S. Blin et al., "Reduced thermal sensitivity of a fiber optic Gyroscope using an air-core photonic bandgap fiber," JLT 25(3), 861-865 (2007).

[4] F. Couny et al., "Reduction of Fresnel back-reflection at splice interface between hollow core PCF and SMF," PTL 19, 1020-1022 (2007).

[5] J. W. Nicholson et al., "Low-loss, low return-loss coupling between SMF and singlemode, hollow-core fibers ," CLEO'14, JTu4A.71.

[6] Y. Jung et al., "Compact few-mode fiber collimator and associated optical components for mode division multiplexed $~, "$ OFC'16, W3D.5.

[7] Y. Jung et al., "Compact 32-core multicore fibre isolator for high density spatial density multiplexed transmission," ECOC'16, W2.B4.

[8] V. A. J. M. Sleiffer et al., "High capacity mode division multiplexed optical transmission in a novel 37-cell ," JLT 32(4), 854-863 (2014).

[9] P. Hofmann et al., "Detailed investigation of mode-field adapters utilizing multimode interference in ," JLT 30(14), 2289-2298 (2012). 